\title{
Implicaciones en Salud Pública de Staphylococcus aureus Meticilino Resistente Adquirido en la Comunidad en Bogotá, Colombia
}

\section{Community-acquired methicillin-resistant Staphylococcus aureus in} Bogotá, Colombia: Public Health implications

\author{
Jorge Alberto Cortes ${ }^{1,2}$, Carlos Andrés Gómez ${ }^{2}$, Sonia Isabel Cuervo ${ }^{1,3}$, \\ Aura Lucía Leal ${ }^{4}$ y GREBO ${ }^{2}$
}

\author{
1 Departamento de Medicina, Facultad de Medicina, Universidad Nacional de Colombia. \\ jorgecortes@yahoo.com, cagomezal@unal.edu.co, sicuervom@unal.edu.co \\ 2 Grupo para el Control de la Resistencia Bacteriana en Bogotá. \\ 3 Grupo de Infectología, Instituto Nacional de Cancerología. \\ 4 Departamento de Microbiología, Universidad Nacional de Colombia. allealc@unal.edu.co \\ Recibido 8 Noviembre 2006/Enviado para Modificación 18 Junio 2007/Aceptado 19 Agosto 2007
}

\section{RESUMEN}

Objetivo Determinar la prevalencia de Staphylococcus aureus resistente a meticilina y sensible a eritromicina y clindamicina (perfil de resistencia sugestivo de ser adquirido en la comunidad) (SARM-AC), de aislamientos ambulatorios de la base de datos de Grebo durante el periodo 2001-2005.

Materiales y Métodos La base de datos del grupo Grebo se integró desde 2001 con más de 22 hospitales participantes en Bogotá. A partir de esta base de datos y con la ayuda del programa Whonet 5.3, se determinaron los perfiles de resistencia de los aislamientos ambulatorios de S.aureus. Se tomaron los puntos de corte establecidos por la CLSI (2003).

Resultados Se identificaron 2308 aislamientos de S. aureus provenientes de aislamientos ambulatorios, de los cuales $618(26,8 \%)$ eran Staphylococcus aureus meticilino resistente (SARM). Setenta y cuatro (3,2 \%) de éstos, presentaban sensibilidad a todos los otros antibióticos (eritromicina, clindamicina), sugestivos de corresponder al fenotipo SARM-AC.

Conclusiones La presencia en Bogotá de aislamiento de $S$. aureus sugestivos de tener el perfil de SARM -AC, implica un gran problema de salud pública debido a su posibilidad de diseminación en la comunidad y cambios en el empleo de antibióticos en los servicios de urgencias. Los datos de nuestro estudio sugieren que los aislamientos de SARM-AC en nuestro medio, pueden ser más comúnes de lo que pensamos.

Palabras Clave: Staphylococcus aureus, Resistencia a la meticilina, Bogotá (fuente: DeCS, BIREME). 


\section{ABSTRACT}

Objective Determining the prevalence of methicillin-resistant Staphylococcus aureus (CA-MRSA) and susceptibility to erythromycin and clindamycin (resistance profile suggestive of being CA-MRSA) in community isolates from de GREBO's database from 2001-2005.

Materials and Methods GREBO's database has been complied since 2001, using more than 22 hospitals in Bogota. S. aureus resistance profiles were determined from community isolates. Whonet 5.3 software and CLSI breakpoints (2003) were used.

Results 2308 S. aureus community isolates were identified. $618(26,8 \%)$ were methicillin-resistant Staphylococcus aureus (MRSA). 74 (3,2 \%) MRSA exhibited susceptibility to all other antibiotics (erythromycin, clindamycin), suggesting that $\mathrm{S}$. aureus belonged to CA-MRSA.

Conclusions CA-MRSA might have emerged in Bogotá thereby having a profound implication for public health due to possible dissemination in the community and because antibiotic protocols for emergency settings should be changed. The results of our study suggested that CA-MRSA could be more common in Bogotá than currently expected.

Key Words: Staphylococcus aureus, methicillin resistance, community-acquired infection (source: MeSH, NLM).

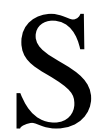

taphylococcus aureus resistente a meticilina adquirido en la comunidad (SARM-AC) ha emergido durante los últimos años como un microorganismo de gran importancia en países desarrollados, en aislamientos de pacientes sin ningún nexo con los servicios hospitalarios(1). En Bogotá, se ha informado de la presencia de casos de SARM-AC en pacientes sin ningún antecedente hospitalario (2). En este estudio, analizamos los patrones de susceptibilidad a los antibióticos de aislamientos ambulatorios de S. aureus de los años 2001 a 2005 de la base de datos del Grupo para el Control de la Resistencia Bacteriana en Bogotá (GREBO) para determinar aquellos con perfil sugestivo de SARM- AC.

\section{MATERIALES Y MÉTODOS}

Hospitales participantes

Se recolectó la información procedente de pacientes atendidos en los siguientes hospitales e Instituciones participantes (2001-2005): Universidad Nacional de Colombia, Hospital Simón Bolívar, Hospital Universitario de San Ignacio, Hospital San José, Hospital Santa Clara, Fundación Hospital San Carlos, Hospital Militar Central, Hospital Universitario Clínica San Rafael, Fundación Cardioinfantil, Instituto Nacional de 
Cancerología, Clínica del Niño, Clínica San Pedro Claver, Hospital de Kennedy, Hospital de la Misericordia, Clínica del Occidente, Clínica Jorge Piñeros Corpas, Saludcoop, Fundación Santa Fe de Bogotá, Clínica Infantil Colsubsidio, Hospital El Tunal, Centro Policlínico del Olaya, Hospital Universitario La Samaritana, Instituto Nacional de Salud, Asociación Colombiana de Infectología, Secretaría Distrital de Salud de Bogotá Recolección de datos

Los laboratorios de microbiología enviaron los informes, los cuales fueron incorporados a una base de datos creada en el software Whonet como se ha descrito en detalle previamente (3).

Los aislamientos de los hospitales participantes se clasificaron en salas de tratamiento intrahospitalario (pisos), UCI y tratamiento extrahospitalario (urgencias, consulta externa, etc). En este caso se analizó sólo la información referente a los pacientes extrahospitalarios.

Datos de susceptibilidad antimicrobiana

La información de los resultados de susceptibilidad antimicrobiana fueron clasificados como sensibles, intermedios o resistentes de acuerdo a los puntos de corte utilizados para cada antibiótico determinados a partir de las recomendaciones del CLSI (Clinical Laboratory Standards Institute) (2003). Se incluyeron los resultados de un aislamiento procesado por cada paciente en cada laboratorio durante cada mes. No se incluyeron aislamientos repetidos.

Para el análisis se considero como posible fenotipo de SARM-AC, los que tenían resistencia a oxacilina (MIC $\geq 4$ ), con sensibilidad a eritromicina (MIC $\leq 0,5$ ) y clindamicina (MIC $\leq 0,5)$. Para determinar la frecuencia de los distintos perfiles de resistencia se utilizó la función "perfiles de resistencia" del programa Whonet. Se excluyeron las cepas que no tenían resultado de susceptibilidad a ninguno de los antibióticos (oxacilina, eritromicina, clindamicina).

Control de calidad Microbiológica

Los laboratorios incluidos en el programa Whonet, fueron sometidos a un programa de calidad externo, llevado a cabo por el laboratorio de Microbiología del Instituto Nacional de Salud.

\section{RESULTADOS}

Se analizaron 316239 perfiles de susceptibilidad disponibles en la base de datos de GREBO, pertenecientes a las instituciones participantes en el período de estudio. Entre 2001 y 2005, fueron identificados un total de 30645 
aislamientos de S. aureus. De éstos, 2308 (7,5\%) provenían de pacientes ambulatorios. El 71,4 \% de los aislamientos ambulatorios fueron susceptibles a oxacilina (S. aureus sensible a meticilina, SASM). El 58,3\% de los aislamientos fueron susceptibles a todos los antibióticos, representando estos a los $S$. aureus multisensibles adquirido en la comunidad. Se obtuvieron 618 aislamientos de SARM que correspondía a 26,8\% del total. Dentro de estos 618, 74 aislamientos (3,2 \% del total) presentaban solo resistencia a oxacilina por lo cual eran sospechosos de ser compatibles con el perfil de SARM-AC. En total 507 (22 \%) SARM tenían perfil de multiresistencia (Tabla 1).

Tabla 1. Perfiles de susceptibilidad de los $S$. aureus en pacientes ambulatorios

\begin{tabular}{lr}
\multicolumn{2}{c}{$(2001-2005)$} \\
\hline \multicolumn{1}{c}{ Tipo de Aislamien to } \\
\hline S. aureus con uno o más antibióticos no probados & \multicolumn{1}{c}{$42(1,8)$} \\
\hline SASM & $1648(71,4)$ \\
Sensible a Clindamicina y Eritromicina & $1345(58,3)$ \\
Resistente a Clindamicina & $46(2)$ \\
Resistente a Eritromicina & $174(7,5)$ \\
Resistente a Clindamicina y Eritromicina & $74(3,6)$ \\
\hline SARM & $618(26,8)$ \\
Sensible a Clindamicina y Eritromicina (SARM-AC $)$ & $74(3,2)$ \\
Resistente a Clindamicina & $22(1)$ \\
Resistente a Eritromicina & $15(0,6)$ \\
Resistente a Clindamicina y Eritromicina & $507(22)$ \\
\hline Totql & $2308(100)$ \\
\hline
\end{tabular}

* Estos aislamientos por present ar resistencia a meticilina y sensibilidad a clindamicina y eritromicina son sugestivos de ser SARM $\quad$-AC. ${ }^{* *}$ Estos aislamientos son considerados también como multiresistentes y por lo tanto sospechosos de ser hospitalarios (SARM-HA).

Las muestras a partir de las cuales se identificaron los 74 aislamientos con el perfil sugestivo de SARM-AC fueron secreciones (45\%) (que incluye muestras de piel y tejidos blandos), sangre (9\%) y orina $6 \%$. Otros tipos de muestra correspondían a esputos, heridas quirúrgicas, aspirados traqueales, faringe, mucosas y representan cada uno menos del $5 \%$ del total.

Durante todo el periodo del estudio la prevalencia de SARM osciló entre $15,5 \%$ y $32 \%$ de los aislamientos ambulatorios. Los aislamientos compatibles con SARM-AC, presentaron durante el periodo de estudio porcentajes de aparición entre 2,2 \% y 4,8 \%.

En general, los aislamientos de SARM y SARM-AC se mantuvieron dentro de un rango estable en todo el periodo de tiempo de estudio, sin evidenciarse brotes (Figura 1). 


\section{DISCUSIÓN}

Los aislamientos de SARM-AC, comenzaron a surgir a principios de 1980 en Australia (4). Durante la última década, la prevalencia de SARM-AC ha crecido, especialmente en E.E.U.U (1). Estos aislamientos de SARM-AC, suponen una gran amenaza para la salud pública, debido a los perfiles de resistencia que exhiben, los factores de virulencia que poseen y el riesgo latente de convertirse en la cepa de $S$. aureus más común en los aislamientos de la comunidad (5). Esto implicaría un gran costo epidemiológico y, por supuesto económico, además del impacto sobre el uso de antibióticos en pacientes ambulatorios.

Figura 1. Prevalencia (\%) de SARM y compatibles com SARM-AC en 2308 aislamientos ambulatorios. Bogotá, 2001-2005

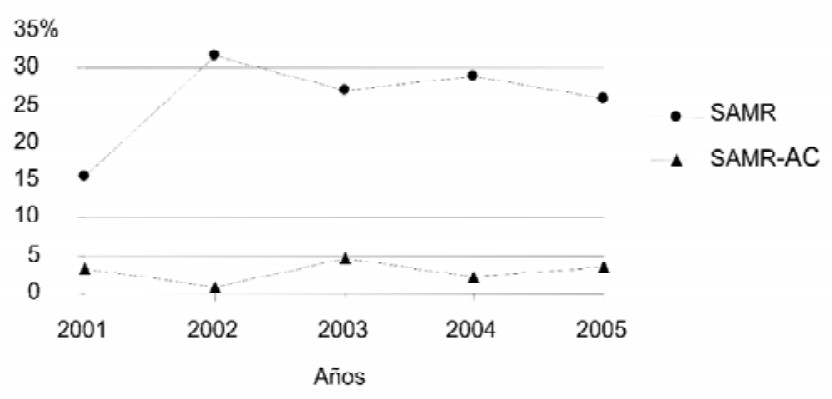

Existen claras diferencias entre los aislamientos de SARM- AC y los adquiridos en el hospital (5). Estas diferencias consisten en una conformación genética distinta, que trae consigo diferentes perfiles de resistencia antibiótica y factores de virulencia característicos(6). También existen también diferencias respecto al espectro clínico de infecciones que producen y el tipo de pacientes que afectan. Un estudio hecho en EE.UU. (7), en el año 2000, demostró estas diferencias. Los pacientes con aislamientos SARM-AC eran en general más jóvenesy se relacionaron con infecciones de piel y tejidos blandos. Además, SARM-AC fue más susceptible a 3 antibióticos distintos a los ß- lactámicos (eritromicina, clindamicina, quinolonas)(8). La anterior descripción soporta que en nuestro estudio hayamos escogido a los aislamientos de $S$. aureus resistentes a oxacilina pero sensibles a clindamicina y eritromicina como aquellos sugestivos de ser SARM-AC.

Nuestro estudio tiene limitaciones, es retrospectivo a partir de una base de datos que está hecha con énfasis en la vigilancia de la resistencia nosocomial y 
no de pacientes ambulatorios. Esto explica el bajo porcentaje de pacientes ambulatorios. Adicionalmente carecemos de la información necesaria para establecer correctamente los SARM-AC y diferenciarlos de aquellos de origen hospitalario, por lo que no podemos comparar los resultados obtenidos en este estudio con los informados en la literatura americana.

Los resultados de este estudio tienen muchas consecuencias tanto en salud pública, como en el campo de vigilancia de la resistencia antibiótica y en las políticas de uso de antibíoticos en servicios de urgencias y consulta externa. En EE.UU, un estudio reciente encontró que la prevalencia de aislamientos de SARM-AC en pacientes que consultaron a urgencias en ciudades distintas, por infecciones de piel y tejidos blandos correspondía a 59 \% (9). Estos hallazgos lo catalogan como el agente más común de este tipo de infecciones en pacientes ambulatorios en EE.UU, tan solo 15 años después de haberse descrito el primer aislamiento en ese país. En Uruguay, el primer reporte de SARM-AC ocurrió en 2001. En los siguientes años, se presentaron en la zona urbana de Montevideo, más de 1000 casos SARM-AC (10). Hasta ahora en nuestro medio se han descrito los dos primeros casos de SARM-AC. Los aislamientos identificados en Bogotá (2), demostraron tener las características genéticas usuales de los SARM-AC de EE.UU. Estas similitudes sugieren que en nuestro medio se pueden estar identificando verdaderos aislamientos de SARM-AC, así como sucedió en EE.UU, en la década de los noventa. Se deben establecer programas de viglancia activa para este microorganismo, y para el personal médico que se enfrenta a este tipo de infecciones en los servicios de urgencias, es necesario reconocer el tipo de manifestaciones clínicas y el espectro de pacientes afectados por SARM-AC, con el fin de sensibilizarlos de la importancia de obtener un diagnóstico microbiológico y de conocer los perfiles de susceptibilidad antimicrobiana en el laboratorio *

Agradecimientos. Agradecemos al Dr. Giancarlo Buitrago, por su aporte en la actualización y facilitación de la base de datos de GREBO.

\section{REFERENCIAS}

1. Nicola Z, Francis JS, E Nuermberger EL, Bishai WR. Community-acquired methicillinresistant Staphylococcus aureus: an emerging threat. Lancet Infect Dis 2005; 5:275-86.

2. Alvarez CA, Barrientes OJ, Leal AL, Contreras GA, Barrero L, Rincón S, et al. Community- associated Methicillin -resistant Staphylococcus aureus, Colombia. Emerg Infect Dis 2006; 12:2000-1. 
3. Leal AL, Eslava J, Alvarez C, Buitrago G, Méndez M, GREBO. Canales endémicos y marcadores de resistencia bacteriana en Instituciones de tercer nivel de Bogotá, Colombia. Rev Salud Publica 2006; 8 (suppl 1): 59-70.

4. Maguire GP, Arthur AD, Boustead PJ, Dwyer B, Currie BJ. Clinical experience and outcomes of community-acquired and nosocomial methicillin-resistant Staphylococcus aureus in a northern Australian hospital. J Hosp Infect 1998;38: 273-81.

5. Wisplinghoff H, Rosato AE, Enright MC, et al. Related clones containing SCCmec type IV predominate among clinically significant Staphylococcus epidermidis isolates. Antimicrob Agents Chemother 2003; 47:3574-9.

6. Fey PD, Said-Salim B, Rupp ME. Comparative molecular analysis of community or hospital-acquired methicillinresistant Staphylococcus aureus. Antimicrob Agents Chemother 2003;47:196-203.

7. Naimi TS, LeDell KH, Como-Sabetti K, et al. Comparison of community and health care-associated methicillin-resistant Staphylococcus aureus infection. JAMA 2003; 290: 2976-84.

8. Salgado CD, Farr BM, Calfee DP. Community-Acquired Methicillin-Resistant Staphylococcus aureus: A Meta-Analysisof Prevalence and Risk Factors. Clin Infect Dis 2003; 36:131-9.

9. Moran GJ, Krishnadasan A, Gorwitz RJ, Fosheim GE, McDougal LK, Carey RB, et al. Methicillin-Resistant S. aureus Infections among Patients in the Emergency Department. N Engl J Med 2006; 355: 666-74.

10. Xiao X. M, Galiana A, Pedreira W, Mowszowicz M, Christophersen I, Machiavello S, et al. Community acquired Methicillin-resistant Staphylococcus aureus, Uruguay. Emerg Infect Dis. 2005: 11(6); 973-977. 